\title{
Gelatinous Ascites: A Cytohistologic Study of Pseudomyxoma Peritonei in 67 Patients
}

\author{
Stephanie L. Jackson, M.D., Ronald A. Fleming, PhD., Brian W. Loggie, M.D., Kim R. Geisinger, M.D., \\ Department of Pathology (SLJ, KRG), Wake Forest University Baptist Medical Center, Winston-Salem, \\ North Carolina; Clinical Research Program (RAF), Glaxo Wellcome, Inc., Research Triangle Park, North \\ Carolina; and University of Texas Southwestern Medical Center (BWL), Moncrief Cancer Center, Fort \\ Worth, Texas
}

Background: Pseudomyxoma peritonei (PMP) is a rare condition characterized by gelatinous ascites. Although the histologic attributes of PMP have been well studied, the cytologic features remain ill defined. Methods: We reviewed the peritoneal washings (PW) in 67 patients with PMP to identify cytomorphologic features useful in classifying cases as either disseminated peritoneal adenomucinosis (DPAM) or peritoneal mucinous carcinomatosis (PMCA). Histologic specimens were correlated with the cytologic diagnoses. Correlation between cytologic diagnosis and patient outcome was investigated. Results: Neoplastic epithelial cells were identified in 63 of 67 PW (94\%). Concordance with the histologic diagnosis was obtained in 61 of 63 cases. Of these $36.5 \%$ were cytologically classified as DPAM with primary appendiceal neoplasms in 19 cases. Thirty-four of 63 cases $(53.9 \%)$ were cytologically diagnosed as PMCA based on PW cytology. Most were of appendiceal or colonic origin. Four cases displayed cytologic features of both DPAM and PMCA. Two discordant cases each with a cytologic diagnosis of PMCA had an appendiceal adenoma. Acellular mucin alone was identified in the PW in four cases. Analysis of follow-up data revealed that cases diagnosed as DPAM had a better prognosis than those diagnosed as PMCA. Conclusions: Cytomorphologic features of epithelial cells in PW material can accurately categorize cases of PMP as either DPAM or PMCA. Furthermore, this categorization appears to have important prognostic implications.

Copyright () 2001 by The United States and Canadian Academy of Pathology, Inc.

VOL. 14, NO. 7, P. 664, 2001 Printed in the U.S.A.

Date of acceptance: March 10, 2001.

Address reprint requests to: Kim R. Geisinger, M.D., Department of Pathology, Wake Forest University Baptist Medical Center, Medical Center Boulevard, Winston-Salem, NC 27157; e-mail: kgeis@wfubmc.edu; fax: 336-716-7595.
KEY WORDS: Pseudomyxoma peritonei, Disseminated peritoneal adenomucinosis, Peritoneal mucinous carcinomatosis, Gelatinous ascites, Mucinous ascites Peritoneal cytology.

Mod Pathol 2001;14(7):664-671

Pseudomyxoma peritonei (PMP) is a condition characterized by the accumulation of mucinous material in the peritoneal cavity (gelatinous ascites). Several theories have been proposed to explain the pathogenesis of PMP including multifocal neoplasia of the peritoneum, ovaries, and appendix (1), mucinous metaplasia of the peritoneum (2), and metastasis, rupture, or leakage from a primary mucinous neoplasm of the ovary or appendix (3-8). Recent studies incorporating immunohistochemical stains such as cytokeratins (CK) 7, 17, 18, and 20, carcinoembryonic antigen (CEA), and HAM-56 and use of genetic analysis by polymerase chain reaction techniques have added strong support to the interpretation that almost all cases of PMP originate from primary appendiceal neoplasms (9-14) Sporadic cases associated with tumors of the breast (15), gallbladder and bile duct (16), pancreas (17), stomach and intestines $(18,19)$, lung $(20)$, and urinary bladder (18) have been reported.

In a pivotal study, Ronnett et al. (21) suggested that the term PMP, to date, has been used inappropriately to describe a clinical entity arising from a morphologically heterogeneous group of benign and malignant pathologic lesions. They proposed abandoning PMP as a histologic diagnosis with separation of the clinical entity of GA into two histologic categories, disseminated peritoneal adenomucinosis (DPAM) and peritoneal mucinous carcinomatosis (PMCA) based on the histologic appearance of the neoplastic epithelium of the peritoneal lesions and the underlying primary tumor. Cases diagnosed as DPAM demonstrated abundant mucin containing scant strips of mucinous epithelium with minimal to moderate cytologic atypia and rare mitoses. The glandular epithelium was 
simple and nonstratified to focally proliferative. Cases diagnosed as PMCA had more abundant epithelium demonstrating marked cytologic atypia. This epithelium formed malignant glands or nests or occurred as individual malignant cells including signet ring cells. An intermediate category was also utilized to indicate specimens that demonstrated histologic features of both DPAM and PMCA as well as cases showing discordant features (21). These cases were diagnosed as PMCA based on the finding of at least focal areas with more typical carcinomatous epithelium. Most cases with dual histologic features were derived from well-differentiated appendiceal or intestinal mucinous adenocarcinomas. Clinically, cases of DPAM were associated with superficial implants, rare parenchymal or nodal involvement and a more benign or indolent course characteristic of the clinical syndrome of PMP. Cases of PMCA were associated with more extensive lymph node involvement, parenchymal invasion, and metastases outside the abdominal cavity. The value of the categorization proposed by Ronnett was reflected in a statistically significant difference in survival between patients with DPAM, and PMCA (21).

Other notable findings in this study included the following: 1) histologically identical cases of DPAM were associated with a variety of appendiceal neoplasms; 2) prognosis could be predicted from the appearance of the peritoneal lesions alone in cases with unknown primaries; 3) most recurrent lesions displayed the same histologic features seen in the primary tumor (DPAM only rarely progressed to PMCA); 4) in all cases of DPAM and PMCA with unknown primary tumors, the appendix was abnormal; 5) no cases of unequivocal ovarian origin were identified; and 6) the clinical condition of GA was almost always associated with a primary tumor of the GI tract (21).

Although the histologic features of PMP have been well studied, the cytologic features remain ill defined (22-30). We reviewed the cytologic features of peritoneal washings (PW) collected intraoperatively and the corresponding histology in 67 cases of PMP to 1) identify cytomorphologic features useful in distinguishing between DPAM and PMCA $(21)$; 2) determine the sites of origin of DPAM and PMCA; and 3) evaluate the prognosis of DPAM and PMCA.

\section{MATERIALS AND METHODS}

Cytologic smears from PW and corresponding histologic specimens from 67 patients clinically diagnosed with PMP were retrieved from the files of the Department of Pathology at Wake Forest University Baptist Medical Center. A small subset of these patients has previously been reported (31). PW fluid was collected intraoperatively and smears of the gelatinous material were fixed in $95 \%$ ethanol for Papanicolaou staining. In 56 of 67 cases, material was also available for cell blocks; it was fixed in $10 \%$ buffered formalin, embedded in paraffin, sectioned, and stained with hematoxylin and eosin. Histopathologic specimens were fixed in $10 \%$ buffered formalin, processed routinely, and stained with hematoxylin and eosin.

All cytologic smears and corresponding cell blocks were examined in a blinded fashion by one of the authors (KRG). Based on cytologic features alone, one of four diagnoses was made: 1) acellular mucin only; 2) DPAM; 3) PMCA; or 4) features of both DPAM and PMCA. Cytologic features that were specifically evaluated included presence or absence of glandular epithelium, size and arrangement of cellular groups, variation in cell size and shape, nuclear size and shape, nuclear chromatin distribution, hyperchromasia, presence or absence of nucleoli, nuclear-to-cytoplasmic ratio, presence or absence of mitoses and single neoplastic epithelial cells and the presence of extracellular mucin. The cytomorphologic features useful for diagnosing DPAM were variably sized, cohesive clusters or monolayered (honeycomb) sheets of cells with discrete cell borders, uniform small, round nuclei with smooth nuclear membranes and inconspicuous nucleoli. Mitotic figures and individual malignant neoplastic cells were not identified. Features for the cytologic diagnosis of PMCA included individual cells, small rounded three-dimensional clusters, or occasional, irregular sheets of cells with enlarged, overlapping nuclei with irregular nuclear membranes, irregular chromatin distribution, variably sized nucleoli, and malignant signet ring cells. Mitotic features were identified in some cases of PMCA (see Fig. 1).

After the cytologic diagnosis was established, the histologic sections were reviewed to determine the categorization of the peritoneal lesion (DPAM versus PMCA) using the criteria established by Ronnett et al. (21). The histologic specimens were also examined to determine the site(s) of origin of PMCA and DPAM. In women in whom a primary tumor could not be identified and cases in which only an ovarian tumor was identified, immunohistochemical staining for CK7 and 20 was selectively applied to peritoneal implants and the ovarian tumor, when available, to determine the site of origin $(9,10)$. Positive staining for both CK7 and 20 or CK7 positivity with negative staining for CK20 was considered consistent with origin from a primary ovarian tumor. Positive staining for CK20 and negative staining for CK7 was considered consistent with origin from a GI (appendiceal or colonic) mucinous neoplasm (9). 


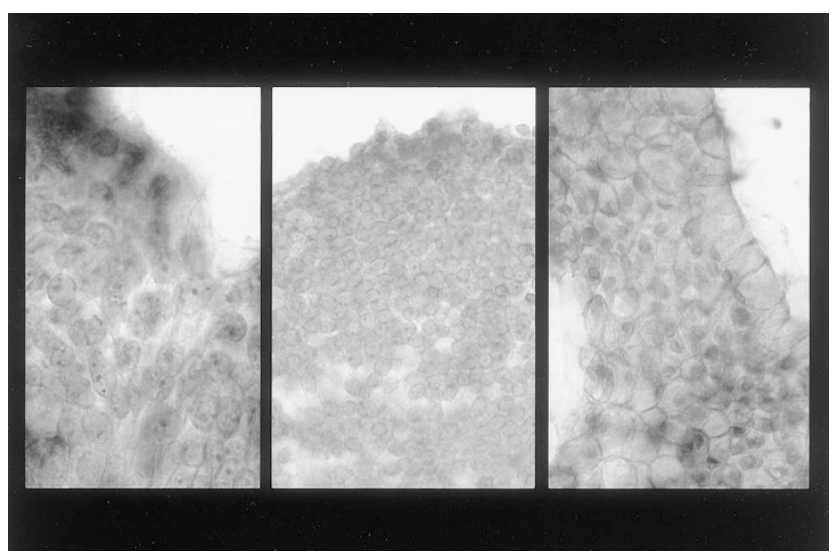

FIGURE 1. Comparison of peritoneal mucinous carcinomatosis (PMCA), low-grade peritoneal mucinous carcinomatosis, and disseminated peritoneal adenomucinosis (DPAM) cytology. PMCA showing marked increase in nuclear to cytoplasmic ratio, nuclear overlap, irregular cell borders, and large, irregular nucleoli (left). Intermediate or low-grade PMCA showing an irregular group border, increased N/C, nuclear crowding and overlap, and occasional, small nucleoli (middle). DPAM showing a smooth border, obvious mucin production, discrete cell borders with small nuclei without nucleoli or nuclear overlap (right).

\section{Antibodies and Immunostaining:}

Information regarding antibodies used in this study including working dilutions and sources is summarized in Table 1. Five-micron-thick sections were obtained from formalin-fixed, paraffinembedded tissue blocks. Immunohistochemical staining with monoclonal antibodies against CK7 and 20 and polyclonal anti-CEA antibodies was performed on the Ventana ES automated stainer (Ventana Medical Systems, Tucson, AZ) by using the enzyme-labeled avidin-biotin method after digestion with pronase. Immunohistochemical staining with polyclonal antibodies against calretinin was performed using the Zymed second generation LAB-SA detection system (Zymed Laboratories, Inc., San Francisco, CA) by using heat induced epitope retrieval and enzyme-labeled streptavidinbiotin amplification. Immunoreactivity was scored semiquantitatively as follows: $1+$ ( 1 to $25 \%$ positive); $2+$ (26 to $75 \%$ positive); $3+$ (76 to $100 \%$ positive).

\section{RESULTS}

Sixty-seven cases of clinically diagnosed PMP included 32 women $(47.7 \%)$ and 35 men $(52.2 \%)$. The ages of the female patients at the time of diagnosis ranged from 24 to 89 years (mean, 56); the ages of the male patients ranged from 24 to 70 years (mean, 53). The presenting signs and symptoms were known in 47 patients: increased abdominal girth (24 patients), abdominal pain (16 patients), abdominal mass/hernia (5 patients), urinary frequency (1 patient), and constipation (1 patient). The majority of patients were treated with extensive tumor debulking followed by cytoreductive surgery with intraoperative intraperitoneal hyperthermic chemotherapy with Mitomycin C. Adjuvant systemic chemotherapy with a variety of agents (5Fluorouracil, cisplatin, Taxol, CPT 11, and Topotecan) was used in a minority of patients. This small group of patients generally consisted of cases of PMCA of nonappendiceal origin and cases of recurrent or progressive disease (DPAM or PMCA) in which therapy with intraperitoneal hyperthermic chemotherapy was ineffective. Clinicopathologic findings are given in Tables 2 and 3.

Of the men, eight (23\%) of 35 cases were classified as DPAM on the basis of the PW cytology. All but one had an appendiceal mucinous (ApM) adenoma. Twenty-two cases $(63 \%)$ were diagnosed as PMCA on the basis of PW cytology. Of these, eight cases (36\%) had an ApM adenocarcinoma and six cases (27\%) had mucinous adenocarcinoma of the large intestine. One case had a mucinous adenocarcinoma of the small bowel, one had a gastroesophageal junctional adenocarcinoma, one had a gastric adenocarcinoma, and one case had a pancreatic primary tumor. Four (30.7\%) with PMCA did not have a known primary site. One case had cytologic features of both DPAM and PMCA. Histologic examination in this case revealed an ApM adenoma and a separate mucinous adenocarcinoma arising in the cecum. Two $(8.7 \%)$ discordant cases occurred, each with a cytologic diagnosis of PMCA but with histology showing an ApM adenoma. Two (5.7\%) specimens that contained only extracellular mucin were diagnosed as PMCA on tissue sections and had an ApMadenocarcinoma.

Of the women, 15 of 32 cases (46.8\%) were classified as DPAM based on PW cytology. Of these, 12 (80\%) had documented ApMadenomas and, of these, 10 exhibited synchronous bilateral ovarian mucinous (OvM) tumors. One patient with an ApMadenoma showed superficial involvement of the

TABLE 1. Antibodies Used

\begin{tabular}{|c|c|c|c|}
\hline Antibody to: & $\begin{array}{c}\text { Clonal } \\
\text { Designation }\end{array}$ & $\begin{array}{l}\text { Working } \\
\text { Dilution }\end{array}$ & Source \\
\hline Cytokeratin 7 & OV-TL 12/30 & $1: 10$ & DAKO, Carpinteria CA \\
\hline Cytokeratin 20 & $\mathrm{~K}_{\mathrm{S}} 20.8$ & $1: 2000$ & DAKO \\
\hline Carcinoembryonic antigen & Polyclonal & $1: 25$ & Biomeda, Foster City, CA \\
\hline Calretinin & Polyclonal & Prediluted & Zymed, San Francisco, CA \\
\hline
\end{tabular}


TABLE 2. Clinicopathologic Findings for Female Patients

\begin{tabular}{|c|c|c|c|c|c|c|}
\hline Case No. & Age (yr) & Cytologic Diagnosis & Appendix & Ovary & Other Primary & Follow-Up (mo) \\
\hline 1 & 56 & DPAM & Adenoma & $\mathrm{MT} / \mathrm{BO}$ & & ANED $(59)^{b, h}$ \\
\hline 2 & 48 & DPAM & Adenoma & $\mathrm{MT} / \mathrm{BO}$ & & ANED $(57)^{b}$ \\
\hline 3 & 44 & DPAM & Adenoma & MT/RO & $\begin{array}{l}\text { LO/ } \\
\text { Muc.Cystadeno-CA } \\
\text { with lung met }{ }^{b}\end{array}$ & $\operatorname{ANED}(53)^{b, d}$ \\
\hline 4 & 72 & DPAM & Adenoma & $\mathrm{MT} / \mathrm{BO}$ & & $\operatorname{ANED}(51)^{b}$ \\
\hline 5 & 46 & DPAM & Adenoma & $\mathrm{MT} / \mathrm{BO}$ & & ANED $(26)^{b}$ \\
\hline 6 & 52 & DPAM & Cystadenoma & $\mathrm{MT} / \mathrm{BO}$ & & $\operatorname{ANED}(11)^{b}$ \\
\hline 7 & 89 & DPAM & Cystadenoma & $\mathrm{MT} / \mathrm{BO}$ & & DNED $(13)^{b}$ \\
\hline 8 & 65 & DPAM & NA & NA & GI primary $^{a}$ & $\operatorname{ASD}(232)^{b}$ \\
\hline 9 & 51 & DPAM & NA & NA & GI primary $^{a}$ & $\operatorname{ASD}(212)^{g}$ \\
\hline 10 & 40 & DPAM & Cystadenoma & $\mathrm{MT} / \mathrm{BO}$ & & $\operatorname{ASD}(110)^{c}$ \\
\hline 11 & 38 & DPAM & Serosal mucin & NA & GI primary $^{a}$ & $\operatorname{ASD}(27)^{b, f}$ \\
\hline 12 & 41 & DPAM & Cystadenoma & $\mathrm{MT} / \mathrm{BO}$ & & $\operatorname{ASD}(7)^{b}$ \\
\hline 13 & 57 & DPAM & Adenoma & $\mathrm{MT} / \mathrm{BO}$ & & $\operatorname{APD}(54)^{d, c, e}$ \\
\hline 14 & 47 & DPAM & Adenoma & $\mathrm{MT} / \mathrm{BO}$ & & $\operatorname{APD}(27)^{b}$ \\
\hline 15 & 46 & DPAM & Adenoma & $\mathrm{MT} / \mathrm{BO}$ & & LTF \\
\hline 16 & 49 & DPAM \& PMCA & $\begin{array}{l}\text { WD Mucinous } \\
\text { Adenocarcinoma }\end{array}$ & MT/RO & & $\operatorname{ANED}(41)^{b, h}$ \\
\hline 17 & 37 & PMCA \& DPAM & $\begin{array}{l}\text { WD Mucinous } \\
\text { Adenocarcinoma }\end{array}$ & $\mathrm{MT} / \mathrm{BO}$ & & $\operatorname{ASD}(26)^{c, h}$ \\
\hline 18 & 29 & DPAM \& PMCA & $\begin{array}{l}\text { WD Mucinous } \\
\text { Adenocarcinoma }\end{array}$ & $\mathrm{MT} / \mathrm{BO}$ & & DOD $(25)^{b, h, k, n}$ \\
\hline 19 & 48 & PMCA & SRCA & Met SRCA/BO & & $\operatorname{APD}(20)^{i}$ \\
\hline 20 & 69 & PMCA & Normal & Met CA/BO & Endocervical CA & $\operatorname{APD}(13)^{d}$ \\
\hline 21 & 36 & PMCA & SRCA & Met SRCA/BO & & $\operatorname{APD}(8)^{i}$ \\
\hline 22 & 62 & PMCA & NA & Met CA/BO & Gastric CA & $\operatorname{APD}(6)^{b}$ \\
\hline 23 & 74 & PMCA & Normal & NA & Colon CA & $\operatorname{APD}(5)^{b}$ \\
\hline 24 & 37 & PMCA & NA & NA & Colon CA & DOD $(19)^{b, h, k}$ \\
\hline 25 & 24 & PMCA & NA & Met SRCCA/BO & Gastric SRCCA & $\operatorname{DOD}(13)^{c}$ \\
\hline 26 & 36 & PMCA & NA & Met CA/BO & Gastric SRCCA & DOD $(12)^{b, h, k, l, m}$ \\
\hline 27 & 27 & PMCA & NA & Met CA/BO & Colon CA/SRCCA & DOD $(10)^{b, h}$ \\
\hline 28 & 54 & PMCA & NA & Met CA/BO & Gastric SRCCA & DOD $(8)^{b, h}$ \\
\hline 29 & 62 & PMCA & Normal & Met CA/BO & GI primary $^{a}$ & $\mathrm{LTF}^{b}$ \\
\hline 30 & 31 & PMCA & Serosal CA & Met CA/BO & GI primary $^{a}$ & $\mathrm{LTF}^{b}$ \\
\hline 31 & 59 & Acellular & Serosal mucin & $\mathrm{MT} / \mathrm{BO}$ & GI primary ${ }^{a}$ & $\mathrm{LTF}^{b}$ \\
\hline 32 & 39 & Acellular & NA & Met CA/RO & Colon CA & $\operatorname{APD}(22)^{b, h, j}$ \\
\hline
\end{tabular}

${ }^{a}$ Consistent with GI primary based on immunohistochemical staining patterns (CK20+; CK7-).

${ }^{b}$ Intraperitoneal hyperthermic chemotherapy with Mitomycin-C (IPHC); ${ }^{c} \mathrm{IPHC} \times 2 ;{ }^{d}$ Taxol and Cisplatin; ${ }^{e} \mathrm{CPT}-11$ and Topotecan; ${ }^{f} \mathrm{CPT}-11$ and Xeloda; ${ }^{g}$ Xeloda and Taxol; ${ }^{h}$ 5-Fluorouracil; ${ }^{i}$ Tumor debulking only; ${ }^{j} \mathrm{CPT}-11 ;{ }^{k}$ IV Mitomycin C; ${ }^{l}$ Etoposide; ${ }^{m}$ VP-16; ${ }^{n}$ Streptozocin.

PMCA, peritoneal mucinous carcinomatosis; SRCA, signet ring cell adenocarcinoma; CA, adenocarcinoma; NA, no tissue available; BO, bilateral ovaries; RO, right ovary; Met, metastatic; MT, mucinous tumor; ANED, alive with no evidence of disease; ASD, alive with stable disease; APD, alive with progressive disease; DNED, no evidence of disease at time of death; LTF, lost to follow up.

right ovary by tumor as well as a well-differentiated mucinous cystadenocarcinoma of the left ovary, which subsequently resulted in pulmonary metastases. Epithelium within the peritoneal implants in this case stained positive for CK20 and negative for CK7 consistent with origin from a GI primary. Three cases of DPAM with unknown primary tumor sites showed positive staining for CK20 and negative staining for CK7 consistent with origin from a GI primary tumor. Of two $(8.3 \%)$ cases with extracellular mucin and no neoplastic epithelium, one was diagnosed as DPAM on tissue sections and had bilateral OvM tumors, which stained positive with CK20 and negative with CK7, consistent with a GI origin. The second acellular cytology specimen had a tissue diagnosis of PMCA with origin from a documented colonic mucinous adenocarcinoma. Twelve (37.5\%) cases were classified as PMCA based on PW cytology. Seven patients had bilateral OvM tumors with primary adenocarcinomas of appendix (2), stomach (4), and endocervix (1). Two cases with bilateral OvM tumors demonstrated positive staining for CK20 and negative staining for CK7 consistent with an appendiceal origin. Three cases had colonic primaries. Three specimens $(4.7 \%)$ had cytologic features of both DPAM and PMCA. In all three cases, histologic sections showed a welldifferentiated mucinous adenocarcinoma of the appendix arising in a mucinous adenoma and bilateral OvM tumors.

Overall, neoplastic epithelial cells were identified in 63 of 67 cases (94\%) of the PW. Concordance with the histologic diagnosis was obtained in 61 of 63 cases $(96.8 \%)$. Of the specimens containing neoplastic cells, 23 of 63 cases (36.5\%) were classified as DPAM with primary ApMneoplasms identified in 19 cases $(82.6 \%)$. Three cases of DPAM, which occurred in women, showed immunohistochemical staining patterns consistent with origin from an appendiceal primary. Thirty-four of 63 cases (54\%) were diagnosed as PMCA based on PW cytology with 10 cases $(29.4 \%)$ of appendiceal origin, nine 


\begin{tabular}{|c|c|c|c|c|c|}
\hline Case No. & Age (yr) & Cytologic Diagnosis & Appendix & Other Primary & $\begin{array}{l}\text { Follow-Up } \\
\text { (mo) }\end{array}$ \\
\hline 1 & 64 & DPAM & Adenoma & & $\operatorname{ANED}(51)^{b}$ \\
\hline 2 & 49 & DPAM & Adenoma & & ANED $(26)^{b}$ \\
\hline 3 & 24 & DPAM & Adenoma & & ANED $(10)^{b}$ \\
\hline 4 & 34 & DPAM & Adenoma & & ANED $(9)^{b}$ \\
\hline 5 & 46 & DPAM & Adenoma & & $\operatorname{ASD}(48)^{b, d, e}$ \\
\hline 6 & 49 & DPAM & Adenoma & & $\operatorname{DOD}(16)^{c, d, g}$ \\
\hline 7 & 67 & DPAM & Adenoma & & $\operatorname{DOD}^{a}(1)^{b}$ \\
\hline 8 & 52 & DPAM & NA & NA & $\mathrm{LTF}^{b}$ \\
\hline 9 & 69 & DPAM \& PMCA & Adenoma & CA/Cecum & DOD@(64) $)^{b, e}$ \\
\hline 10 & 39 & PMCA & Normal & CA/Cecum & $\operatorname{ANED}(13)^{b}$ \\
\hline 11 & 51 & PMCA & Adenocarcinoma & & ANED $(11)^{b}$ \\
\hline 12 & 69 & PMCA & $\begin{array}{r}\text { Well-differentiated } \\
\text { adenocarcinoma }\end{array}$ & & $\operatorname{ASD}(53)^{b, d}$ \\
\hline 13 & 45 & PMCA & Adenocarcinoma & & APD $(84)^{c, d}$ \\
\hline 14 & 53 & PMCA & Adenocarcinoma & & APD $(39)^{c, d, f}$ \\
\hline 15 & 37 & PMCA & Normal & CA/Colon & APD $(27)^{b, d}$ \\
\hline 16 & 53 & PMCA & Adenocarcinoma & & $\operatorname{APD}(13)^{b}$ \\
\hline 17 & 54 & PMCA & NA & CA/unknown primary & $\operatorname{APD}(12)^{b}$ \\
\hline 18 & 47 & PMCA & Adenocarcinoma & & $\operatorname{APD}(11)^{b, d, e}$ \\
\hline 19 & 62 & PMCA & Normal & CA/Colon & $\operatorname{APD}(3)^{b}$ \\
\hline 20 & 55 & PMCA & Adenocarcinoma & & DOD $(19)^{b, e}$ \\
\hline 21 & 39 & PMCA & NA & CA/unknown primary & DOD $(12)^{c}$ \\
\hline 22 & 56 & PMCA & NA & SRCA/unknown primary & DOD (8) \\
\hline 23 & 54 & PMCA & NA & CA/Small bowel & $\operatorname{DOD}(7)^{b, d}$ \\
\hline 24 & 52 & PMCA & NA & CA/Pancreas & DOD $(6)^{b}$ \\
\hline 25 & 70 & PMCA & NA & CA/unknown primary & DOD $(4)^{b}$ \\
\hline 26 & 34 & PMCA & NA & CA/Colon & $\operatorname{DOD}(3)^{b, d}$ \\
\hline 27 & 35 & PMCA & NA & $\begin{array}{l}\text { CA/Gastroesophageal } \\
\text { junction }\end{array}$ & $\operatorname{DOD}(3)^{b, d}$ \\
\hline 28 & 60 & PMCA & NA & CA/Gastric & $\operatorname{DOD}(2)^{b}$ \\
\hline 29 & 63 & PMCA & NA & CA/Colon & $\operatorname{DOD}(2)^{b, d}$ \\
\hline 30 & 70 & PMCA & Adenocarcinoma & & $\mathrm{LTF}^{b}$ \\
\hline 31 & 51 & PMCA & NA & CA/Cecum & LTF \\
\hline 32 & 56 & Acellular & Adenocarcinoma & & $\operatorname{ANED}(77)^{b}$ \\
\hline 33 & 64 & Acellular & Adenocarcinoma & & $\operatorname{ANED}(15)^{b, d}$ \\
\hline 34 & 71 & PMCA & Adenoma & & APD $(116)^{b}$ \\
\hline 35 & 64 & PMCA & Adenoma & & $\operatorname{ASD}(12)^{b}$ \\
\hline
\end{tabular}

${ }^{a}$ Death due to complications of therapy; ${ }^{b}$ Intraperitoneal hyperthermic chemotherapy with Mitomycin-C (IPHC); ${ }^{c}$ IPHC $\times 2$; ${ }^{d} 5$-Fluorouracil; ${ }^{e}$ CPT- $11 ;{ }^{f}$ Xeloda; ${ }^{g}$ Cytoxan and Cisplatin.

DPAM, disseminated peritoneal adenomucinosis; PMCA, peritoneal mucinous carcinomatosis; SRCA, signet ring cell adenocarcinoma; NA, No tissue available; ASD, alive with stable disease; ANED, alive with no evidence of disease; DOD, died of disease; LTF, lost to follow up; APD, alive with progressive disease.

(26.4\%) tumors of colonic origin, five cases $(14.7 \%)$ with gastric primaries, and 1 case each from gastroesophageal, small bowel, pancreatic and endocervical primaries. Two cases in women showed immunohistochemical staining patterns consistent with origin from a GI primary, and four (11.7\%) cases had an unknown primary tumor. Four cases (6.3\%) had cytologic features of both DPAM and PMCA. Histologic sections in three of these showed a well-differentiated adenocarcinoma of the appendix arising in a mucinous adenoma. The fourth case had a mucinous adenoma of the appendix and a separate, well-differentiated mucinous adenocarcinoma arising in the cecum. Two discordant $(2.9 \%)$ cases each with a cytologic diagnosis of PMCA had histology showing an ApMM adenoma. Acellular mucin alone was identified in four cases $(5.9 \%)$, one of which was diagnosed histologically as DPAM of appendiceal origin, and three of which were diagnosed as PMCA two from an appendiceal primary and the other from a colonic primary.
In all cases containing epithelial cells and diagnosed as DPAM, uniform neoplastic cells occurred in flat sheets with smooth contours. Cell borders were discrete, and there were low nuclear to cytoplasmic (N/C) ratios. Nuclear membranes were smooth and nucleoli were absent or inconspicuous. Nuclear overlap was not a feature of DPAM. No mitoses were identified. Compared with cases diagnosed as PMCA, cases of DPAM had larger and more numerous epithelial clusters, whereas single neoplastic cells were not identified. Thus, it appears as if cohesion is better preserved in adenoma cells relative to frankly malignant glandular cells. In contrast to cases of DPAM, cases of PMCA were more heterogeneous cytologically. Epithelial clusters tended to be smaller, fewer in number, and most had a three-dimensional configuration, as compared with the flat sheets seen in DPAM. Hyperchromatic, obviously malignant nuclei with clumped chromatin and prominent, occasionally multiple nucleoli were seen in some cases. Other cases demonstrated only indi- 
vidual malignant signet ring cells or individually dispersed cells with eccentric, enlarged hyperchromatic nuclei, prominent nucleoli, clumped chromatin, irregular nuclear membranes and delicate pale cytoplasm often containing vacuoles. In occasional cases, the neoplastic cells in PMCA occurred in flat monolayered sheets.

Compared with those of DPAM cases, these sheets were smaller, had irregular outlines, showed obvious nuclear overlap, elevated N/C ratios, and most cells contained small to prominent nucleoli. These cases were diagnosed as PMCA but had cytologic features that suggested a lower grade lesion than cases having overtly malignant features. Despite this finding, no distinction was made between low and high-grade lesions. All were called PMCA.

Mesothelial cells were usually easily identified. However, in occasional cases, the identification of cells as being mesothelial in origin rather than epithelial was difficult if not impossible. When cell block material was available, immunohistochemical staining with CEA and calretinin was used to make this distinction. When the distinction could not be made with some degree of certainty, based on cytologic features or using immunohistochemistry, the cells were considered mesothelial in origin.

\section{DISCUSSION}

PMP is a term originally used to describe the clinical syndrome of GA associated with a ruptured cystadenoma of the ovary (32). In 1901, Fraenkel first associated PMP with a ruptured mucocele of the appendix (33). Since then, a heterogeneous group of mucinous tumors, both benign and malignant, from various sites have been associated with the syndrome (15-20). As a result, PMP has been considered a slowly progressive disease associated with frequent recurrences and an unpredictable prognosis.

Recently, Ronnett et al. (21) suggested that the term PMP be used only as a clinical descriptor for GA. They proposed a new classification system based on the morphologic features of the epithelium identified in peritoneal implants and on the nature of the underlying lesion. The category designated "disseminated peritoneal adenomucinosis" described lesions containing abundant extracellular mucin associated with simple to focally proliferative epithelium with minimal cytologic atypia and mitotic activity. The vast majority of these cases were attributed to ruptured appendiceal adenomas. The category of "peritoneal mucinous carcinomatosis" described peritoneal lesions also containing abundant extracellular mucin but with more abundant epithelium that showed sufficient cytologic atypia and architectural complexity to warrant a diagnosis of carcinoma. Most of these cases were attributed to GI malignancies. A third category, termed "peritoneal mucinous carcinomatosis with intermediate or discordant features" was used to describe cases that showed predominantly features of DPAM but also had focal areas of carcinoma. These cases were often associated with welldifferentiated mucinous adenocarcinomas of the appendix. The discordant category described cases that had carcinomatous peritoneal lesions associated with appendiceal adenomas that did not show carcinomatous transformation.

Using this histologic classification system, clinical and prognostic differences were noted. DPAM was clinically characterized by superficial, noninvasive peritoneal implants and only rarely with parenchymal or nodal involvement. No cases were associated with extra-abdominal metastases. In contrast, invasive peritoneal implants, parenchymal invasion, lymph node metastases and metastases outside the abdominal cavity characterized PMCA. Prognostically, a marked stratification was noted in the survival rates among the categories. Age-adjusted 5-year survival for the DPAM category was $43 \%$, that for PMCA was $7 \%$ and the intermediate/discordant category had a rate of $38 \%$ (21).

Although the histologic features of PMP have been well studied, the cytologic features remain ill defined. Recent cytologic studies, of small series, have emphasized the usefulness of neoplastic epithelial cells, reactive mesothelial cells, acellular mucin, histiocytes and spindle cells in making the diagnosis of PMP (22-30). However, few reports detail the cytomorphologic features of the epithelial cells in GA. We identified acellular mucin, reactive mesothelial cells and histiocytes in most of our cases but, similar to Shin et al. (25) we identified epithelial cells in the majority of specimens.

Using the histologic classification system of Ronnett et al. (21) as a template, we evaluated the cytologic features of epithelial cells in PW from patients diagnosed clinically with PMP. Based on our set of cytologic features, we divided cases into DPAM and PMCA. Intermediate cases with features of both DPAM and PMCA were identified, as well as, discordant cases in which the cytologic features were not in concordance with the histologic findings. Similar to the findings of Ronnett et al. (21), we noted prognostic and behavioral differences between the groups.

Most cases of DPAM were attributed to an appendiceal adenoma. These cases showed more abundant epithelium in cytologic preparations when compared with cases of PMCA, presumably because of the superficial nature of the implants in DPAM as opposed to the invasive implants in PMCA. In women who had mucinous ovarian tu- 
mors but in whom the appendix was not available, immunohistochemical staining of peritoneal and ovarian lesions with CK7 and 20 all showed patterns consistent with origin from an appendiceal primary (9). No cases of unequivocal ovarian origin were identified. Peritoneal implants were noninvasive and parenchymal and nodal involvement were rare.

Most cases of PMCA were associated with an identifiable appendiceal or GI mucinous adenocarcinoma. Again, peritoneal implants in women with mucinous ovarian tumors for whom no appendix was available showed immunohistochemical staining patterns consistent with a gastrointestinal origin. No cases of PMCA were of ovarian origin. Peritoneal implants were invasive and often associated with parenchymal invasion and lymph node metastases.

Four cases demonstrated features of both DPAM and PMCA. These patients appeared to have a prognosis intermediate between DPAM and PMCA although the small number of cases limits significant evaluation. Acellular specimens diagnosed histologically as either DPAM or PMCA seem to have a slightly better prognosis than cellular specimens in the same histologic category although the number of cases is too small to draw any firm conclusions. Also similar to findings of Ronnett (21), in cases with multiple recurrences, the cytologic features of the epithelium did not appear to change over time.

Some authors suggest that the presence of epithelial cells outside the appendix alone is sufficient for the diagnosis of malignancy (34-36). In this report we assume, as other authors have $(21,37$, 38), that PMP may result from both benign and malignant primary lesions and that the associated epithelial cells within the GA fluid may also be either benign (DPAM) or malignant (PMCA). For the diagnosis of adenocarcinoma arising in the appendix, we required the presence of cytologically malignant glands, nests or single cells invading the appendiceal wall and showing a desmoplastic response. Epithelial cells, nests and glands within the appendiceal wall or within peritoneal implants alone were insufficient for a diagnosis of carcinoma.

Early cytologic reports proposed that the condition of PMP could be rendered by virtue of the background material of mucin, mesothelial cells and reactive fibroblasts, in smears from ascitic fluid $(24,28)$. Other reports suggested that the presence of epithelial cells in peritoneal fluid may be associated with a worse prognosis or higher recurrence rates that acellular specimens (23). Based on our data, we propose that the presence of epithelial cells alone is not prognostically significant in cases of GA. Instead, it is the cytologic classification of the epithelial cells as either adenomatous (DPAM) or carcinomatous (PMCA) that is prognostically signif- icant. This categorization results in two distinct categories of disease, each associated with different biologic behaviors and prognosis. We also noted that epithelial cells were identified in $94 \%$ of cases, and concordance with the histologic diagnosis was attained in $97 \%$ of cases. Thus, PW cytology can effectively be used, as an aid in the correct categorization of PMP as either DPAM or PMCA, and this categorization appears to have prognostic implications. When comparing this study with others on the subject, the prevalence of epithelial cells in our study may be a reflection of the specimen collection method, namely peritoneal washing and not spontaneous ascitic fluids used in other studies.

\section{REFERENCES}

1. Seidman JD, Elsayed AM, Sobin LH, Tavassoli FA. Association of mucinous tumors of the ovary and appendix. A clinicopathologic study of 25 cases. Am J Surg Pathol 1993;17: 22-34.

2. Sandenbergh HA, Woodruff JD. Histogenesis of pseudomyxoma peritonei: review of 9 cases. Obstet Gynecol 1977; 49:339-45.

3. Campbell JS, Low P, Ferguson JP, Krongold I, Kemeny T, Mitton DM, et al. Pseudomyxoma peritonei et ovarii with occult neoplasms of the appendix. Obstet Gynecol 1973;42: 897-902.

4. Fernandez RN, Daly JM. Pseudomyxoma peritonei. Arch Surg 1980;115:409-13.

5. Ghosh BC, Huvos A, Whitlet HW. Pseudomyxoma peritonei. Dis Colon Rectum 1972;15:420-5.

6. Limber GK, King RE, Silverberg SG. Pseudomyxoma peritonei: a report of 10 cases. Ann Surg 1973;178:587-93.

7. Smith JW, Kemeny N, Caldwell C, Banner P, Sigurdson E, Huvos A. Pseudomyxoma peritonei of appendiceal origin. The Memorial Sloan-Kettering Cancer Center experience. Cancer 1992;10:396-410.

8. Young RH, Gilks CB, Scully RE. Mucinous tumors of the appendix associated with mucinous tumors of the ovary and pseudomyxoma peritonei. A clinicopathological analysis of 22 cases supporting an origin in the appendix. Am J Surg Pathol 1991;15:415-29.

9. Wang NP, Zee S, Zarbo RJ, Bacchi CE, Gown AM. Coordinate expression of cytokeratins 7 and 20 defines unique subsets of carcinomas. Appl Immunohistochem 1995;3:99-107.

10. Guerrieri C, Franlund B, Boeryd B. Expression of cytokeratin 7 in simultaneous mucinous tumors of the ovary and appendix. Mod Pathol 1995;8:573-6.

11. Ronnett BM, Shmookler BM, Diener-West M, Sugarbaker $\mathrm{PH}$, Kurman RJ. Immunohistochemical evidence supporting the appendiceal origin of pseudomyxoma peritonei in women. Int J Gynecol Pathol 1997;16:1-9.

12. DeYoung BR, Gaffey MJ, Zarbo RJ, Swanson PE, Mills SE. HAM-56 immunoreactivity in pseudomyxoma peritonei: a potential method of separating ovarian from appendiceal primary lesions. Mod Pathol 1995;8(Suppl.):88A.

13. Szych C, Staebler A, Connolly DC, Wu R, Cho KR, Ronnett BM. Molecular genetic evidence supporting the clonality and appendiceal origin of pseudomyxoma peritonei in women. Am J Pathol 1999;154:1849-55.

14. Cuatrecasas M, Matias-Guiu X, Prat J. Synchronous mucinous tumors of the appendix and the ovary associated with pseudomyxoma peritonei. A clinicopathologic study of six cases with comparative analysis of c-ki-ras mutations. Am J Surg Pathol 1996;20:739-46. 
15. Hawes D, Robinson R, Wira R. Pseudomyxoma peritonei from metastatic colloid carcinoma of the breast. Gastrointest Radiol 1991;16:80-2.

16. Young RH, Scully RE. Ovarian metastases from carcinoma of the gallbladder and extrahepatic bile ducts simulating primary tumors of the ovary. A report of 6 cases. Int J Gynecol Pathol 1990;9:60-72.

17. Chejfec G, Rieker WJ, Jablokow VR, Gould VE. Pseudomyxoma peritonei associated with colloid carcinoma of the pancreas. Gastroenterology 1986;90:202-5.

18. Costa MJ. Pseudomyxoma peritonei. Histologic predictors of patient survival. Arch Pathol Lab Med 1994;202-5.

19. Ikejiri K, Anai H, Kitamura K, Yakabe S, Saku M, Yoshida K. Pseudomyxoma peritonei concomitant with early gastric cancer: report of a case. Surg Today 1996;26:923-5.

20. Kurita M, Komatsu H, Hata Y, Shiina S, Ota S, Terano A, et al. Pseudomyxoma peritonei due to adenocarcinoma of the lung: case report. J Gastroenterol 1994;29:344-8.

21. Ronnett BM, Zahn CM, Kurman RJ, Kass ME, Sugarbaker PH, Shmookler BM. Disseminated peritoneal adenomucinosis and peritoneal mucinous carcinomatosis. A clinicopathologic analysis of 109 cases with emphasis on distinguishing pathologic features, site of origin, prognosis, and relationship to "pseudomyxoma peritonei." Am J Surg Pathol 1995; 19:1390-408.

22. Pfitzer P, Richartz G. Cytology of pseudomyxoma peritonei. Cytopathology 1995;6:304-15.

23. Mulvany N, Ooi K. Pseudomyxoma peritonei: a cytohistopathologic study of 9 cases. Diagn Cytopathol 1996;15: 144-50.

24. Pisharodi L, Bedrossian C, Gudlaugsson E. Cytologic diagnosis of pseudomyxoma peritonei: common and uncommon causes. Int J Surg Pathol 1995;2(Suppl.):10-13.

25. Shin H, Sneige N. Epithelial cells and other cytologic features of pseudomyxoma peritonei in patients with ovarian and/or appendiceal mucinous neoplasms. A study of 12 patients including 5 men. Cancer Cytopathol 1999;87(6):17-23.

26. Costa M, Oertel YC. Cytology of pseudomyxoma peritonei: report of two cases arising from appendiceal cystadenomas. Diagn Cytopathol 1990;6:201-3.

27. Rammou-Kinnia R, Sirmakechian-Karra T. Pseudomyxoma peritonei and malignant mucocele of the appendix: a case report. Acta Cytol 1986;23:169-72.

28. Leiman G, Goldberg R. Pseudomyxoma peritonei associated with ovarian mucinous tumors. Cytologic appearance in five cases. Acta Cytol 1992;36:299-304.

29. Gupta RK, Naran S. Cytodiagnosis of pseudomyxoma peritonei in cases suspected of ovarian tumors. Diagn Cytopathol 1993;9:682-4.

30. Gu M, Zuna RE. Columnar cells in smears from pseudomyxoma peritonei. Diagn Cytopathol 1997;16:182-3.

31. Loggie BW, Fleming RA, Geisinger KR. Assessment of cytology before and after intraperitoneal hyperthermic chemotherapy for peritoneal carcinomatosis. Acta Cytol 1996;40: 1154-8.

32. Werth R. Klinische und anatomische untersuchungen zur lehre von den bauchgeschwuelsten und der laparotomie. Arch Gynaekol 1884; 24: 100-18.

33. Fraenkel E. Ueber das sogennante pseudomyxoma peritonei. Muench Med Wschr 1901;48:965-71.

34. Higa E, Rosai J, Pizzimbono CA, Wise L. Mucosal hyperplasia, mucinous cystadenoma and mucinous cystadenocarcinoma of the appendix. A re-evaluation of appendiceal "mucocele." Cancer 1973;32:1525-41.

35. Gibbs NM. Mucinous cystadenoma and cystadenocarcinoma of the vermiform appendix with particular reference to mucocele and pseudomyxoma peritonei. J Clin Pathol 1983;26:413-21.

36. Wolff M, Ahmed A. Epithelial neoplasms of the vermiform appendix (exclusive of carcinoid): II. Cystadenomas, papillary adenomas and adenomatous polyps of the appendix. Cancer 1976;37:2511-22.

37. Qizilbash AH. Mucoceles of the appendix: Their relationship to hyperplastic polyps, mucinous cystadenomas, and adenocarcinomas. Arch Pathol 1975;99:548-55.

38. Aho AJ, Heinonen R Lauren P. Benign and malignant mucocele of the appendix: Histological types and prognosis. Acta Chir Scand 1973;139:392-400. 